\title{
Erratum to: Quantifying soil hydraulic properties and their uncertainties by modified GLUE method
}

\author{
Yifan Yan, Jianli Liu*, Jiabao Zhang, Xiaopeng Li, and Yongchao Zhao \\ Institute of Soil Science Chinese Academy of Sciences, Nanjing, Jiangsu, China
}

Received March 28, 2017; accepted May 31, 2017

\section{Corrected authors affiliation}

\author{
Yifan Yan ${ }^{1,2}$, Jianli Liu ${ }^{1}$, Jiabao Zhang ${ }^{1}$, Xiaopeng Li $^{1}$, Yongchao Zhao ${ }^{1,2}$ \\ ${ }^{1}$ Institute of Soil Science, Chinese Academy of Sciences, Nanjing 210008, People's Republic of China \\ ${ }^{2}$ University of Chinese Academy of Sciences, Beijing 100049, People's Republic of China
}

\section{Added}

Acknowledgements

This work was financial supported by National Natural Science Foundation of China (41771265) and "135"Plan (Frontier Program) of Insititute of Soil Science, Chinese Academy of Sciences (ISSASIP1661). 